\title{
Ordering tendencies in the binary alloys of Rh, Pd, Ir, and Pt: Density functional calculations
}

\author{
Gus L. W. Hart \\ gus.hart@gmail.com \\ Brian Kolb \\ Stefan Müller \\ David B. Botts
}

Follow this and additional works at: https://scholarsarchive.byu.edu/facpub

Part of the Astrophysics and Astronomy Commons, and the Physics Commons

\section{Original Publication Citation}

Brian Kolb, Stefan Mueller, David Botts and Gus L. W. Hart "Ordering tendencies in the binary alloys of Rh, Pd, Ir, and Pt,"Phys. Rev. B 7414426 (3 Oct. 26). The original article may be found here: http://prb.aps.org/abstract/PRB/v74/i14/e14426

\section{BYU ScholarsArchive Citation}

Hart, Gus L. W.; Kolb, Brian; Müller, Stefan; and Botts, David B., "Ordering tendencies in the binary alloys of Rh, Pd, Ir, and Pt: Density functional calculations" (2006). Faculty Publications. 285.

https://scholarsarchive.byu.edu/facpub/285

This Peer-Reviewed Article is brought to you for free and open access by BYU ScholarsArchive. It has been accepted for inclusion in Faculty Publications by an authorized administrator of BYU ScholarsArchive. For more information, please contact ellen_amatangelo@byu.edu. 


\title{
Ordering tendencies in the binary alloys of $\mathbf{R h}, \mathbf{P d}$, Ir, and Pt: Density functional calculations
}

\author{
Brian Kolb, ${ }^{1}$ Stefan Müller, ${ }^{2}$ David B. Botts, ${ }^{3}$ and Gus L. W. Hart ${ }^{1,4, *}$ \\ ${ }^{1}$ Department of Physics and Astronomy, Northern Arizona University, Flagstaff, Arizona 86011, USA \\ ${ }^{2}$ Lehrstuhl für Festköperphysik, Universität Erlangen-Nürnberg, D-91058 Erlangen, Germany \\ ${ }^{3}$ Department of Chemistry, Northern Arizona University, Flagstaff, Arizona 86011, USA \\ ${ }^{4}$ Department of Physics and Astronomy, Brigham Young University, Provo, Utah 84602, USA
}

(Received 19 October 2005; revised manuscript received 7 September 2006; published 30 October 2006)

\begin{abstract}
The binary alloys of $\mathrm{Rh}, \mathrm{Pd}$, Ir, and Pt are important because of their high catalytic potential. We report in this paper that the $\mathrm{Rh}_{1-x} \mathrm{Ir}_{x}$ and $\mathrm{Rh}_{1-x} \mathrm{Pt}_{x}$ systems, long thought to phase separate at low temperatures, actually exhibit miscibility over the entire concentration and temperature range. We find low critical ordering temperatures, indicating that long-range order is unlikely to be observed experimentally. These results are compared with previous theoretical predictions for the other binary alloys of $\mathrm{Rh}, \mathrm{Pd}$, Ir, and $\mathrm{Pt}$ and with calculations performed here on the $\mathrm{Pt}_{1-x} \mathrm{Ir}_{x}$ and $\mathrm{Pd}_{1-x} \mathrm{Ir}_{x}$ systems. We discuss these results and investigate the mechanisms governing ordering vs phase separating behavior in all six binaries of the Rh-Pd-Ir-Pt quartet. In particular, we examine why, given the chemical similarity of all members of the quartet, three of their binaries (PdIr, PtIr, and $\mathrm{RhPd}$ ) phase separate while the remaining three ( $\mathrm{RhIr}, \mathrm{RhPt}$, and $\mathrm{PdPt}$ ) order.
\end{abstract}

DOI: 10.1103/PhysRevB.74.144206

PACS number(s): 64.75.+g, 61.66.-f

\section{INTRODUCTION}

The binary alloys of the Rh-Pd-Ir-Pd quartet have been misunderstood for nearly half a century. Experiments done by Raub in the 1950s showed that three of these systems (RhPd, PdIr, and PtIr) exhibit miscibility gaps with relatively high critical temperatures. ${ }^{1}$ An observed correlation between critical temperature and the difference in pure-metal melting points prompted arguments that RhIr, PdPt, and RhPt would also phase separate, but with somewhat lower critical temperatures. Experimental verification of this prediction was made difficult by the relatively long equilibration times for these systems.

Via first-principles calculations, we have discovered, however, that the $\mathrm{Rh}_{1-x} \mathrm{Ir}_{x}$ system exhibits an ordering tendency. This result is in direct conflict with the prediction made by Raub and subsequently adopted by most phase diagram compilations. ${ }^{2}$ This discovery, in conjunction with similar, earlier studies for the PdPt and RhPt systems, ${ }^{3,4}$ demands a complete rethinking of the physics and chemistry of Rh-Pd-Ir-Pt binary alloys. A detailed understanding of these systems is of interest because of their catalytic properties and because they form a model system for studying the physical parameters governing ordering behavior. Traditional arguments involving electronegativity differences and lattice mismatch do not apply in this system because of the great similarity of the metal constituents. The deeper investigation required to explain the ordering tendencies in these systems may uncover new understanding of the important physical properties determining ordering behavior.

In this paper, we present our findings on the $\mathrm{Rh}_{1-x} \mathrm{Ir}_{x}$ system. A $(T=0)$ convex hull of stable configurations is predicted based on a converged cluster expansion. Additionally, cluster expansions are constructed for the phase-separating PdIr and PtIr systems. These results are discussed in conjunction with those found previously for the other binaries in the Rh-Pd-Ir-Pt quartet. Various approaches for predicting the ordering behavior in the systems are discussed. Tradi- tional arguments are shown to be insufficient to explain the observed ordering trends and more involved arguments are explored.

\section{COMPUTATIONAL APPROACH}

Total energy calculations were performed with the Vienna ab initio simulation package ${ }^{5,6}$ (VASP) utilizing projector augmented wave (PAW) potentials $\mathrm{s}^{7,8}$ generated under the generalized gradient approximation ${ }^{9,10}$ (GGA). The PAW potentials used for rhodium and palladium treated $4 p$ electrons in the valence. In all calculations, full relaxations were permitted over all internal and external degrees of freedom. Energies were converged to within $0.1 \mathrm{meV} /$ atom. All calculations used an equivalent $k$ point division scheme (of at least $15 \times 15 \times 15$ divisions) to ensure a uniform density $k$ mesh for all structures. ${ }^{11}$ All Bader analysis ${ }^{12,13}$ was performed on fully converged charge densities using at least a $24 \times 24$ $\times 24$ fast Fourier transform grid. Partial core charge corrections were included in the Bader analysis to prevent Bader surface fragmentation.

The precise workings of the cluster expansion method are described in Refs. 14-20 and only a brief description will be given here. At each lattice site in a binary $\mathrm{A}_{1-x} \mathrm{~B}_{x}$ alloy we can define a spin variable $\hat{S}_{i}$ which is +1 if site $i$ is occupied by an $A$ atom and -1 if it is occupied by a $B$ atom. Any configuration-dependent property can then be expressed exactly ${ }^{15}$ as a sum over all lattice interactions

$$
Z=J_{0}+J_{1} \sum_{i} \hat{S}_{i}+\sum_{i, j} J_{i, j} \hat{S}_{i} \hat{S}_{j}+\sum_{i, j, k} J_{i, j, k} \hat{S}_{i} \hat{S}_{j} \hat{S}_{k}+\cdots
$$

where $Z$ is the property of interest, $\hat{S}_{i}= \pm 1$ gives the occupation at lattice site $i$, and the first sum is over all single lattice sites, the second over all pairs of sites, and so on. The problem is simplified somewhat since the expansion coefficients for any symmetry-equivalent interaction are equal for any location on the lattice. The values of the set of expansion 
coefficients $\{J\}$ will, in general, be different for every system and property of interest and must be chosen so that Eq. (1) is an accurate representation of the property $Z$. One way to obtain the expansion coefficients is to perform firstprinciples calculations and fit the calculated values to Eq. (1). Since the actual number of cluster interactions is astronomical for any macroscopic system, Eq. (1) must be truncated in some reasonable manner. In the present work, this was accomplished by utilizing a genetic algorithm to maximize the predictive accuracy of the model with respect to interaction parameters used. ${ }^{21,22}$

Cluster expansions were constructed using 62 input structures for RhIr, 73 input structures for RhPt, and 37 each for PdIr and PtIr. Fitting parameters were chosen to produce the best predictions. Convergence was checked by direct calculation of selected predictions and further via a leave-manyout cross-validation scheme. ${ }^{23-26}$ The small lattice mismatch in these systems (the largest mismatch for any system examined here is $\approx 3 \%$ ) means that explicit inclusion of a constituent-strain cluster expansion is unnecessary.

Order-disorder transition temperatures were calculated via Monte Carlo simulations using the cluster expansion Hamiltonian. Simulated annealing was carried out on a (20 atom $)^{3}$ lattice using 300 spin flips/site/step.

Finally, we acknowledge that $0 \mathrm{~K}$ density functional theory (DFT) calculations are not always reliable, for example, not including the Van der Waals contribution to the binding energy of noble metals, ${ }^{27}$ but such issues are unlikely to play a role in our study as we are looking at differences between calculated energies (which all involve similar atoms). Of the six binaries we discuss, the three that we find to be phase separating have been observed experimentally and there is strong agreement on the short-range order of $\mathrm{PtRh}$ as well. Thus our study agrees well with all the properties of the quartet that are known experimentally.

\section{RESULTS AND DISCUSSION}

\section{A. The $\mathbf{R h}_{1-x} \operatorname{Ir}_{x}$ System}

Following Raub's prediction of phase separation in the $\mathrm{Rh}_{1-x} \mathrm{I} \mathrm{r}_{x}$ system there has been, to our knowledge, no experimental or theoretical work investigating the low-temperature structure of this alloy. Consequently, the assessed $\mathrm{Rh}_{1-x} \mathrm{Ir}_{x}$ phase diagrams still show a miscibility gap with a maximum miscibility-gap temperature of $\approx 1123 \mathrm{~K}$ at $x=\frac{1}{2}$, as predicted by Raub. ${ }^{2}$

To examine the ordering behavior of RhIr at low temperature, total energy calculations were carried out using VASP. From these total energies, the formation enthalpy can be calculated as

$$
\Delta H=E_{A B}-\left[(1-x) E_{A}+(x) E_{B}\right]
$$

where $E_{A}, E_{B}$, and $E_{A B}$ are the free energies per atom of pure $A$, pure $B$, and the $A B$ alloy (at concentration $x$ ), respectively.

All formation enthalpies calculated in this way were negative, indicating ordering rather than phase-separating behavior. Since RhIr was found to order, it is desirable to examine the convex-hull and stable configurations of the system. The

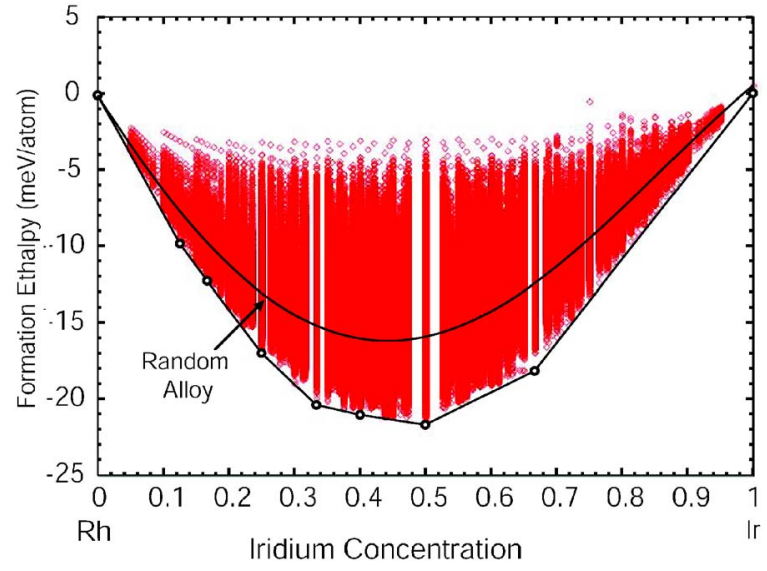

FIG. 1. (Color online) Plot showing direct enumeration search of formation enthalpies. Every configuration with 20 or fewer atoms in the unit cell $\left(\approx 3 \times 10^{6}\right.$ structures $)$ was predicted based on the cluster interaction parameters. Note that all configurations possess a negative formation enthalpy. The dark circles on the convex hull indicate breaking point configurations. Also shown is the random alloy formation enthalpy curve.

high computational cost of quantum-mechanical calculations makes their use in direct enumeration ground-state searches impractical. In light of this, the VASP calculations were coupled with an Ising Hamiltonian in a cluster expansion formulation. ${ }^{14-20}$ Since evaluation of the cluster expansion Hamiltonian for any given configuration is trivial, formation enthalpies for a large number $\left(\approx 3 \times 10^{6}\right.$ in the present case $)$ may be rapidly calculated and a direct enumeration ${ }^{28}$ search for ground-state structures is feasible. Further, since the cluster expansion is directly based on first-principles, quantummechanical input, it correctly includes all quantum effects that were present in the input set and the error in the cluster expansion predictions can be made reasonably small. The cluster expansion constructed here for RhIr has an average prediction error of $\pm 0.6 \mathrm{meV} /$ atom.

Figure 1 shows the predicted formation enthalpies for all configurations up to 20 atoms in the unit cell $\left(\approx 3 \times 10^{6}\right.$ structures). As shown in the figure, RhIr exhibits complete miscibility over the entire range of concentration. The RhIr convex-hull possesses a number breaking points, with a higher concentration of such points on the Rh-rich side. This system is atypical in that almost all of the predicted groundstate structures are relatively large (close to 20 atoms in the unit cell). There are, however, two important points to note about the predicted ground-state line shown in Fig. 1. First, most of the breaking points (the kinks in the convex hull which represent thermodynamically stable structures) are relatively shallow indicating that the order-disorder transition temperatures will probably be low. Second, many of the breaking points have degenerate or nearly degenerate configurations lying slightly above them in energy. The error in the cluster expansion is on the order of the energetic separation of these structures. Thus, while the breaking point concentrations are relatively insensitive to the fit used, the precise structures predicted to be ground states can change somewhat depending on the fit. This ambiguity in the identities of some of the ground-state structures does not pose a 
problem, however, because this system exhibits extremely low critical ordering temperatures-it is extremely unlikely that the $T \rightarrow 0$ ground states will be experimentally realized.

Figure 1 shows that both the formation enthalpies and the enthalpy of mixing (defined as the difference between the energy of the ground-state line and that of the random alloy at a given concentration) are negative, indicating that ordered-structure formation is thermodynamically favorable at $0 \mathrm{~K}$. For any ordering system, there is some temperature at which free-energy lowering by entropic effects overwhelms the chemical contribution and a disordered configuration is favorable. The small magnitudes of the formation and mixing enthalpies hint that this temperature is relatively low for RhIr. This assertion was confirmed by Monte Carlo simulations, which indicate a critical ordering temperature at 50-50 at. \% concentration of $T_{c} \approx 80 \mathrm{~K}$. Such low transition temperatures mean that experimental observation of long-range order is extremely unlikely.

\section{B. The $\mathbf{R h}_{1-x} \mathbf{P t} t_{x}$ System}

Similar to $\mathrm{Rh}_{1-x} \mathrm{Ir}_{x}$, it was postulated for decades that the $\mathrm{Rh}_{1-x} \mathrm{Pt}_{x}$ system shows miscibility gap over the whole concentration regime for temperatures lower than $1030 \mathrm{~K}$ (see e.g., Ref. 29). However, already some years ago, localdensity-approximation-based first-principles studies found exclusively negative formation enthalpies for several ordered compounds. ${ }^{4}$ In order to be consistent with the $\mathrm{Rh}_{1-x} \mathrm{Ir}_{x}$ system, we repeated these DFT calculations now using GGAbased PAW potentials. Indeed, we find negative formation enthalpies for all calculated compounds with values between -2 and $-22 \mathrm{meV} /$ atom, speaking for a weak preference toward ordering.

The enthalpies of the geometrically fully relaxed compounds were then used to construct a cluster expansion as briefly described in Sec. II resulting in a ground-state diagram very similar to that of the $\mathrm{Rh}_{1-x} \mathrm{Ir}_{x}$ system (Fig. 1). For 50-50 concentration, the so-called " $\mathrm{CH}$ " structure (NbP) - a $\mathrm{Rh}_{2} \mathrm{Pt}_{2}$ superlattice along the [201] direction-was found to be the $T=0 \mathrm{~K}$ ground state with a formation enthalpy of $\Delta H=-20.7 \mathrm{meV} /$ atom. This small energy value makes it clear that already at room temperature no long-range order can be expected. In order to study the ordering tendencies as a function of temperature, we used the effective interactions $\{J\}$, Eq. (1), of the system in Monte Carlo simulations. Figure 2 shows the calculated mixing enthalpies over the whole concentration regime for three different temperatures as well as for the random alloy (wide solid line). According to reported phase diagrams, Rh-Pt should still be a solid solution at $T=1100 \mathrm{~K}$ (solid line), while for $T=800$ and $500 \mathrm{~K}$ (dashed lines), a decomposition of the alloy into nearly elemental Pt and Rh crystals should occur. As can be seen, this is not the case: Independent of the temperature, mixing is always energetically preferred over phaseseparation. Therefore, from our calculations we conclude that there exists no miscibility gap in the $\mathrm{Rh}_{1-x} \mathrm{Pt}_{x}$ system.

The result above is manifested by recent diffuse neutron and $\mathrm{x}$-ray diffraction measurements: ${ }^{30}$ This method allows for a determination of short-range order (SRO) parameters

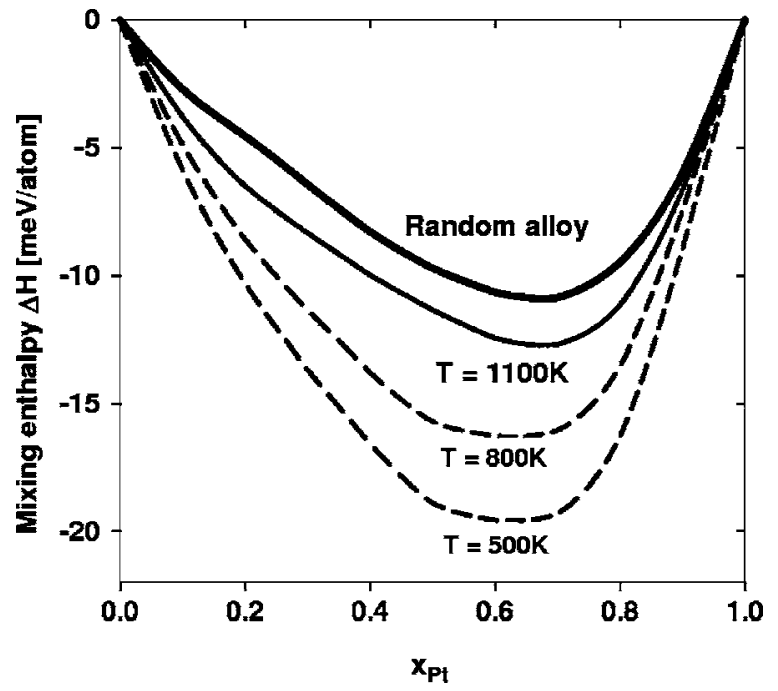

FIG. 2. $\mathrm{Rh}_{1-x} \mathrm{Pt}_{x}$ : Mixing enthalpies as function of concentration. For all temperatures exclusively negative enthalpies are found.

from diffuse intensity patterns in order to quantify the degree of ordering in a solid solution. The Warren-Cowley SRO parameters are given by

$$
\alpha_{j}=1-\frac{P_{A B}^{j}}{2 x_{A} x_{B}} .
$$

The sign of $\alpha_{j}$ indicates whether atoms in a given distance $j$ prefer $A B$ ordering $\left(\alpha_{j}<0\right)$ or clustering $\left(\alpha_{j}>0\right)$. The SRO parameters are normalized such that $-1 \leq \alpha_{j} \leq+1 ; \alpha_{j}=0$ for all $j$ stands for a perfectly random alloy, i.e., an alloy without any atomic correlations. The experimental investigation was carried out for a temperature of $T=1000 \mathrm{~K}$ which would already be in the two-phase region of the phase diagram so that positive values are expected for the SRO parameters. However, this is not the case: The experimental $\alpha_{j}^{\prime}$ 's are all close to zero, which means that the solid solution represents nearly an ideal random alloy. This is in excellent agreement with the energetics of the system as discussed in Fig. 2: For $T=1100 \mathrm{~K}$ the mixing enthalpy of the system is already nearly identical to that of the random alloy. Only the first two neighbors show weak ordering tendencies (Table I): While the nearest-neighbor sites show a small preference for an occupation by unlike neighbors, the opposite is true for the second-neighbor sites.

TABLE I. Comparison between experimental (Ref. 30) and predicted SRO parameter for the first two neighbor distances of a $\mathrm{Rh}_{0.47} \mathrm{Pt}_{0.53}$ alloy $(T=1000 \mathrm{~K})$. The found values are close to zero, indicating that the solid solution comes close to an ideal random alloy.

\begin{tabular}{ccc}
\hline \hline Neighbor & $\alpha^{\text {expt }}$ & $\alpha^{\text {theor }}$ \\
\hline 0 & 1.175 & 1.000 \\
1 & -0.046 & -0.027 \\
2 & 0.017 & 0.047 \\
\hline \hline
\end{tabular}


TABLE II. Phase transition temperatures for all six binary systems in the quartet. For the phase-separating systems, $T_{c}$ refers to the phase separation at the top of the miscibility gap (not necessarily 50-50 at. \% concentration) taken from Refs. 1, 31, and 32. For the ordering systems, $T_{c}$ refers to the predicted order-disorder phase transition at a concentration of $50 \%$. Note that the temperatures for phase separation are much higher than for ordering, and that for the ordering system the temperatures are likely to be overestimated to some degree since the effects of vibrational entropy are not included.

\begin{tabular}{ccc}
\hline \hline System & $T_{c}(\mathrm{~K})$ \\
\hline \multicolumn{3}{c}{ Phase separating } \\
RhPd & 1118 \\
IrPd & 1233 \\
IrPt & 1755 \\
& & \\
& & \\
RhIr & Ordering & 80 \\
RhPt & & 300 \\
$\operatorname{PdPt}$ & & 190 \\
\hline \hline
\end{tabular}

\section{The remaining binaries}

The $\mathrm{Rh}_{1-x} \mathrm{Ir}_{x}$ and $\mathrm{Rh}_{1-x} \mathrm{Pt}_{x}$ systems discussed in the preceding sections are two of six possible binaries in the $\mathrm{Rh}$ -Pd-Ir-Pd quartet. $\mathrm{Rh}_{1-x} \mathrm{Pt}_{x}$ and two of the remaining binaries ( $\mathrm{RhPd}$ and $\mathrm{PdPt}$ ) have been previously studied via the cluster expansion. ${ }^{3,4}$ These cluster expansions demonstrated ordering behavior in $\mathrm{RhPt}$ and $\mathrm{PdPt}$ (ordering at 50 at. \% into $\mathrm{NbP}$ and $L 1_{0}$ phases, respectively) and phase separation in $\mathrm{RhPd}$. These studies also provide us with predicted formation enthalpies for the random alloy. Like RhIr, both RhPt and PdPt possess relatively low critical ordering temperatures $\left(T_{c}=300\right.$ and $190 \mathrm{~K}$, respectively). Table II lists the temperatures at which the order-disorder or phase separation transition occurs for all six systems in the quartet.

To complete the picture, we constructed cluster expansions for the $\mathrm{Pd}_{1-x} \mathrm{Ir}_{x}$ and $\mathrm{Pt}_{1-x} \mathrm{Ir}_{x}$ systems in a manner analogous to that for RhIr and RhPt. The cluster expansions for both systems indicate phase separation at all concentrations, in agreement with experiment.

\section{The full quartet}

Figure 3 summarizes the ordering behavior of each of the six possible binaries in the Rh-Pd-Ir-Pd quartet. Looking at the figure, it is not at all surprising that Raub was unable to correctly predict the ordering behavior of this quartet. He happened to select the three phase-separating systems (indicated by dashed lines) for measurement and then, very reasonably, assumed that the others would follow suit. In fact, given the strikingly similar atomic and crystallographic properties of the members of the quartet (see Table III), it is quite difficult to explain, even retrospectively, the ordering tendencies exhibited by these alloys.

Typical a priori approaches to predict ordering behavior rely on contrasting the properties of the constituent species.

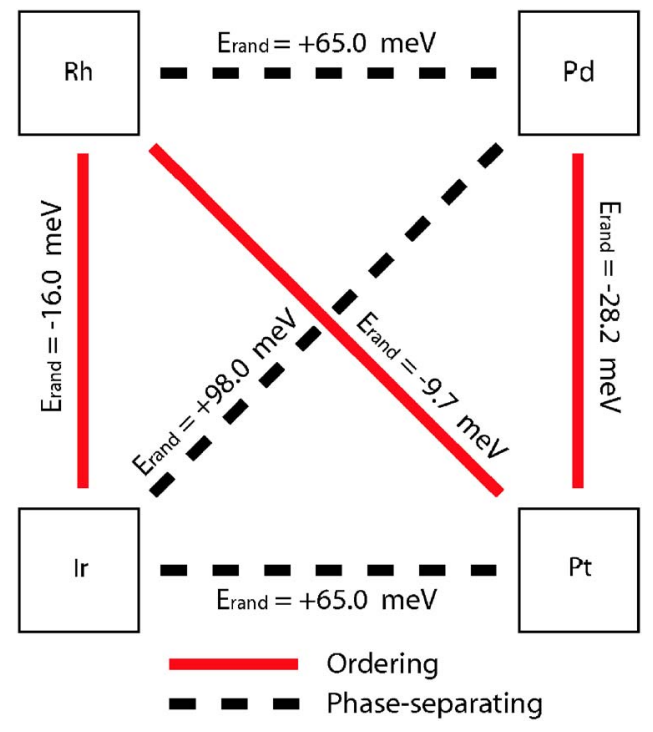

FIG. 3. (Color online) Summary of the ordering behavior of all six binary alloys of $\mathrm{Rh}, \mathrm{Pd}$, Ir, and Pt. Also shown are the formation enthalpies for the random alloy at a 50-50 at. \% concentration, as determined by their respective cluster expansions (energies for the PdPt, RhPd, and RhPt systems are taken from Refs. 3 and 4).

A glance at Table III quickly indicates that finding atomicproperty differences consistent with the ordering tendencies for all six binaries will not be easy. These approaches have been successful in the past and it is useful to ask what they say about the binaries of this quartet. Perhaps the most obvious candidate is electronegativity differences since we know these often control the charge transfer and, hence, the bonding. Simple electronegativity arguments, however, are insufficient to explain the observed trends as some systems with identical electronegativity differences behave in opposite manners. In order to obtain a more realistic assessment of electronegativity (an environment-dependent quantity), Bader analysis was used to calculate charge transfer in several structures of each of the six binaries. There was found no clear correlation between the degree or direction of charge transfer in a system and the sign or magnitude of its formation enthalpy. ${ }^{36}$ Charge density difference $(\Delta \rho)$ calculations (not shown) corroborate the fact that consideration of charge transfer does not produce a consistent picture of the ordering tendencies.

The Hume-Rothery rules predict complete miscibility in all systems of the quartet (a prediction that is only true at elevated temperatures for PdIr, PtrIr, and RhPd, all of which exhibit prominent miscibility gaps). The model of Miedema et $a l .{ }^{37,38}$ predicts ordering in $\mathrm{RhPt}$ and phase separation in the other systems. ${ }^{39}$ Examination of a Pettifor map shows that all six of the binaries fall in a region with a low concentration of ordered structures. ${ }^{40}$ The few surrounding structures are mostly $L 1_{0}$ and $\mathrm{CsCl}$. The pseudopotential radii structure map of Zunger ${ }^{41}$ also places all six binaries in a $\mathrm{CsCl}-L 1_{0}$ region, although this region is much more densely populated than that of the Pettifor map. Of the six binaries only PdPt orders in the $L 1_{0}$ structure at $x=\frac{1}{2}$. Clearly, even these heuristic models fail to produce accurate predictions for the ordering tendencies of this quartet. 
TABLE III. Summary of important atomic and crystallographic properties for the Rh-Pd-Ir-Pt quartet.

\begin{tabular}{ccccc}
\hline \hline Element & $\begin{array}{c}\text { Electron } \\
\text { configuration }\end{array}$ & $\begin{array}{c}\text { Lattice } \\
\text { constant }(\AA)\end{array}$ & $\begin{array}{c}\text { Pauling } \\
\text { (Ref. 33) }\end{array}$ & $\begin{array}{c}\text { Electronegativity } \\
\text { Allred-Rochow } \\
\text { (Refs. 34 and 35) }\end{array}$ \\
\hline $\mathrm{Rh}$ & {$[\mathrm{Kr}] 4 d^{8} 5 s^{1}$} & 3.80 & 2.2 & 1.45 \\
$\mathrm{Pd}$ & {$[\mathrm{Kr}] 4 d^{10}$} & 3.89 & 2.2 & 1.55 \\
$\mathrm{Ir}$ & {$[\mathrm{Xe}] 4 f^{14} 5 d^{7} 6 s^{2}$} & 3.84 & 2.2 & 1.35 \\
$\mathrm{Pt}$ & {$[\mathrm{Xe}] 4 f^{14} 5 d^{9} 6 s^{1}$} & 3.92 & 2.2 & 1.44 \\
\hline \hline
\end{tabular}

Common band-filling arguments also fail to produce consistent predictions of ordering in these systems. In their simplest form, such arguments hold that all alloys composed of late transition metal constituents should phase separate owing to nearly complete population of the valence $d$ bands. Thus, these arguments would hold that all six members of this quartet should phase-separate. Further, as shown in Fig. 4 , there exists no qualitative difference between the siteprojected densities of states for these systems (bandwidths, centers of gravity, and hybridizations are very similar in all cases) and no obvious reason, based on the band picture, that they should behave differently from each other.

Several unexpected cases of ordering in other binaries, particularly those of $3 d-5 d$ and $4 d-5 d$ late transition metals, ${ }^{43-45}$ have been explained in terms of relativistic effects. These arguments hold that relativity is responsible for stabilizing the alloys by a twofold mechanism. First, relativistic contraction of the valence $d$ bands causes a general decrease in the lattice constant. This effect is more pronounced in the $5 d$ metals than in those of higher series. The
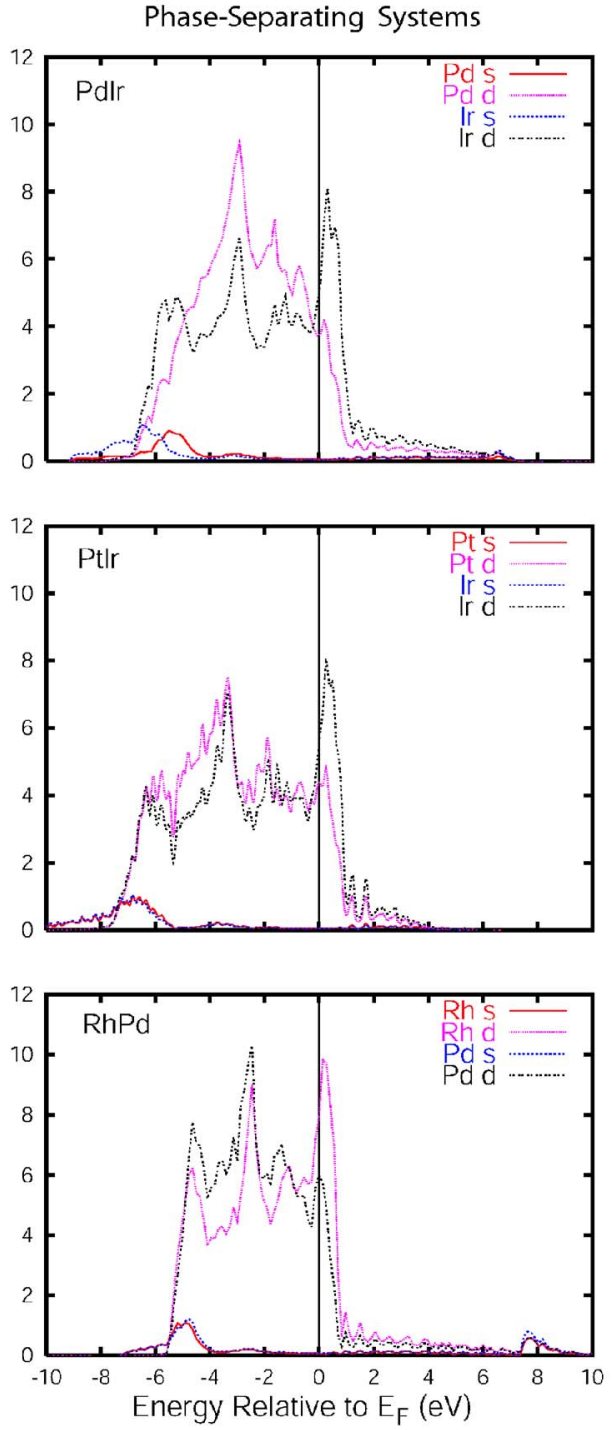

Ordering Systems
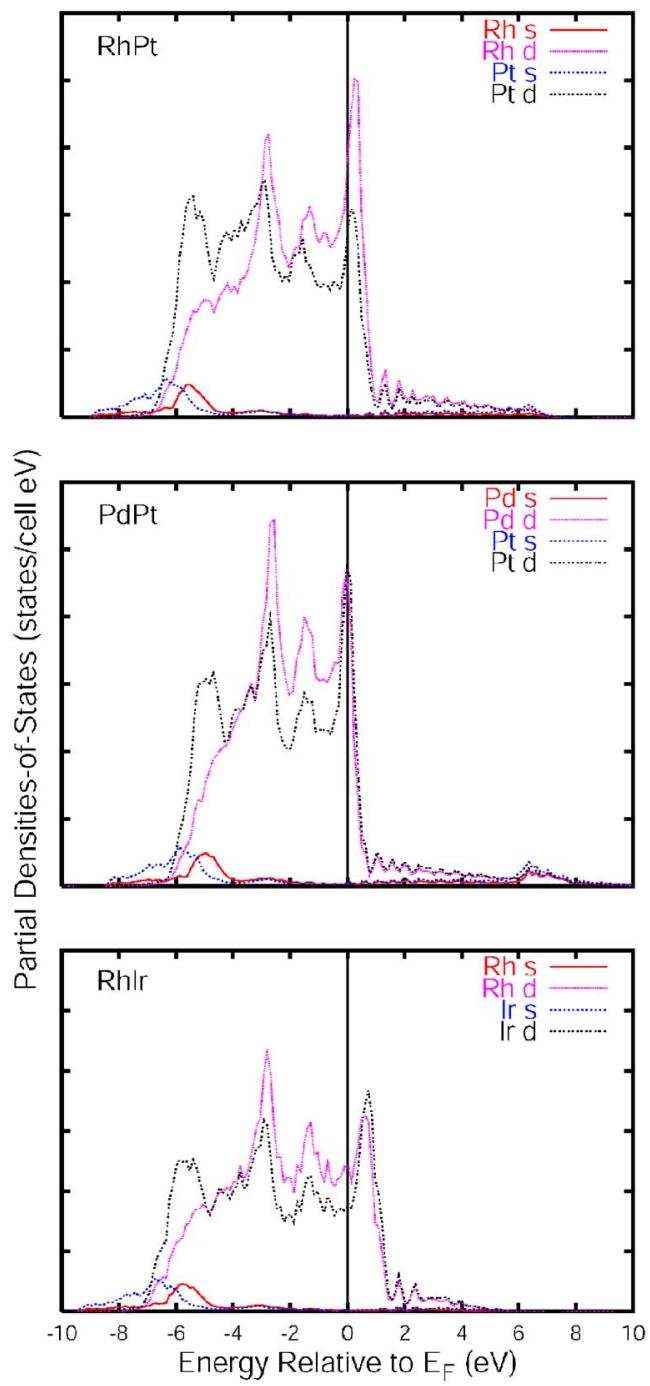

FIG. 4. (Color online) Siteprojected densities of states for each of the six binary alloys of the quartet in the quasirandom atomic configuration (Ref. 42). Shown are the $s$-like and $d$-like states averaged over all atoms of each type within the unit cell. 
TABLE IV. Results of the volume deformation $\left(\Delta E_{\mathrm{vol}}\right)$, chemical $\left(\Delta E_{\text {chem }}\right)$, and relaxation $\left(\Delta E_{\text {relax }}\right)$ energies for the quasirandom structures (Ref. 42). Units are meV/atom.

\begin{tabular}{ccccc}
\hline \hline System & $\Delta H$ & $\Delta E_{\text {vol }}$ & $\Delta E_{\text {chem }}$ & $\Delta E_{\text {relax }}$ \\
\hline PdIr & 89.82 & 9.44 & 90.84 & -10.46 \\
PtrIr & 57.02 & 21.91 & 47.28 & -12.17 \\
RhPd & 59.74 & 15.70 & 55.02 & -10.98 \\
RhPt & -10.91 & 31.09 & -34.24 & -7.77 \\
PdPt & -22.44 & 1.29 & -23.19 & -0.54 \\
RhIr & -17.49 & 2.57 & -18.67 & -1.4 \\
\hline \hline
\end{tabular}

net result is a lowering (possibly) of lattice mismatch and a decrease in the strain energy of the alloy. But this argument cannot apply in our case since the mismatches do not correlate with the predicted ordering.

Second, $d$-band contraction of the $5 d$ metals relative to the $3 d$ and $4 d$ metals reduces the energy gap between atomic $d$ bands and increases their hybridization. The end result is a lowering of the alloy chemical energy by creating and populating low-energy states in preference to those near the Fermi level. Again, this argument is inapplicable ${ }^{46}$ to our case as the hybridization is similar in both the ordering and phase separating systems, as is clear in Fig. 4.

In any alloy system there are two main (and usually competing) factors governing ordering vs phase-separating behavior. Constituent strain is always a positive contribution to the total energy and arises from the fact that the general shape and volume of the alloy are not the same as those for the pure endpoints. Thus, the constituent materials must be distorted from their ideal relative positions in order to form the alloy. Typically, the strain energy is direction dependent and is particularly large at the interface between two pure phases. The second factor is chemical energy resulting from charge rearrangement (i.e., bonding) in the alloy. Allowing charge rearrangement in the system will always lower the free energy of the alloy but does not necessarily mean the free energy is lower than a concentration-weighted average of the pure end points.

To determine which of these factors dominates the ordering behavior in each of the six systems, we can break the formation enthalpies down into physically transparent components. We first define the volume deformation energy $\left(E_{\mathrm{vol}}\right)$ as the energy required to isometrically deform the pure end points from their equilibrium volumes to the volume of the combined alloy. This quantity depends on the bulk moduli of the pure materials and the degree to which they must be distorted to achieve the average volume of the alloy. We next define the chemical energy $\left(E_{\text {chem }}\right)$ as the energy given off when the two pure end points (deformed to the final volume of the alloy) are brought together to form an alloy. Finally, we define the relaxation energy $\left(E_{\text {relax }}\right)$ as the energy released when all internal degrees of freedom of the alloy are allowed to relax. These definitions allow for qualitative comparison between the various components of the formation enthalpy.

Such analysis performed on quasirandom structuresmoderately sized structures chosen such that their cluster- expansion representation is similar to that of a random alloy (see Ref. 47)_yields Table IV. In every case, the sign of the formation enthalpy is the same as the sign of the chemical energy, indicating that the driving force for phase separation in the PdIr, PtrIr, and PdRh alloys is chemical in nature. Strain effects certainly play a role in determining the energies of the various structures, but the chemical energy seems solely responsible for determining alloy ordering.

The lesson learned from this analysis is that the dominating factor in determining ordering or phase-separating behavior in these six binaries is "chemistry." However, taken individually, typical explanations of chemical effects (electronegativities, $d$-band filling, etc.), i.e., typical divisions of the energy, offer an incomplete explanation of the behavior of the quartet. Because of the delicate balance between competing factors, chemical effects must be taken in toto.

\section{CONCLUSION}

We have found that $\mathrm{Rh}_{1-x} \mathrm{Ir}_{x}$ is ordering rather than phase separating. This result is at odds with previous predictions that the system would phase separate around $1150 \mathrm{~K}$. Although we find the $x=0.5$ ground state to be a (311) superlattice, the low calculated transition temperature indicates that long-range order is extremely unlikely to be observed experimentally. The $\mathrm{Rh}_{1-x} \mathrm{Pt}_{x}$ system shows nearly the same behavior as $\mathrm{Rh}_{1-x} \mathrm{Ir}_{x}$ : No hints for the existence of a miscibility gap for temperature below $1030 \mathrm{~K}$ could be found. In contrast to the RhIr system, the "CH" compound ( $\mathrm{NbP}$ ) was found to be the ground state at $x=50 \%$.

There now exist experimental and theoretical data for each of the six binaries of the Rh-Pd-Ir-Pd quartet. We thus attempted to find a consistent theory that was able to reproduce the ordering tendencies of these alloys. Many phenomenological theories were examined such as common electronegativity and band-filling arguments. None of these approaches were found to yield acceptable predictions with respect to the ordering observed in these systems. We found that "chemical" effects dominate the energetics in the quartet and are primarily responsible for the observed ordering is phase-separating trends.

Finally, we believe that the difficulty we encountered in invoking a simple explanation for the behavior of the $\mathrm{Rh}-\mathrm{Ir}$ -Pd-Pt quartet is not unusual. Although an arbitrarily chosen (but conveniently consistent) rule of thumb can always be found to explain the differences between two systems, finding a simple, single, dominating influence that is applicable across a range of compounds will not be possible generally because there is none. Thus, as we have learned from the Rh-Ir-Pd-Pt quartet, one should be skeptical of claims in the literature like "system $A$ orders and $B$ phase separates because of factor $X$." Such implied rules are problematic because (i) there will often be a system $C$ for which factor $X$ would imply behavior like system $A$, yet $C$ behaves like $B$ instead; hence (ii) the importance of factor $X$ in explaining the differences between $A$ and $B$ is anecdotal, rather than definitively causal. 


\section{ACKNOWLEDGMENTS}

This work was supported by the National Science Foundation under Grant No. DMR-0244183 and by Deutsche
Forschungsgemeinschaft under Grant No. Mu1648/2. We thank Volker Blum for stimulating discussions and Alex Zunger for helpful suggestions during the course of this work.
*Corresponding author. Electronic address: gus_hart@byu.edu

${ }^{1}$ E. Raub, J. Less-Common Met. 1, 3 (1959).

${ }^{2}$ Binary Alloy Phase Diagrams, edited by T. B. Massalski, H. Okamoto, P. R. Subramanian, and L. Kacprzak (American Society for Metals, Metals Park, OH, 1990)

${ }^{3}$ Z. W. Lu, S.-H. Wei, and A. Zunger, Phys. Rev. Lett. 66, 1753 (1991)

${ }^{4}$ Z. W. Lu, B. M. Klein, and A. Zunger, J. Phase Equilib. 16, 36 (1995)

${ }^{5}$ G. Kresse and J. Furthmüller, Comput. Mater. Sci. 6, 15 (1996).

${ }^{6}$ G. Kresse and J. Furthmüller, Phys. Rev. B 54, 11169 (1996).

${ }^{7}$ G. Kresse and D. Joubert, Phys. Rev. B 59, 1758 (1999).

${ }^{8}$ P. E. Blöchl, Phys. Rev. B 50, 17953 (1994).

${ }^{9}$ Y. Wang and J. P. Perdew, Phys. Rev. B 44, 13298 (1991).

${ }^{10}$ J. P. Perdew, J. A. Chevary, S. H. Vosko, K. A. Jackson, M. R. Pederson, D. J. Singh, and C. Fiolhais, Phys. Rev. B 46, 6671 (1992).

${ }^{11}$ S. Froyen, Phys. Rev. B 39, 3168 (1989).

${ }^{12} \mathrm{R}$. Bader, Atoms in Molecules: A Quantum Theory (Oxford University Press, New York, 1990).

${ }^{13}$ G. Henkelman, A. Arnaldsson, and H. Jónsson, Comput. Mater. Sci. 36, 354 (2006).

${ }^{14}$ J. M. Sanchez and D. de Fontaine, Phys. Rev. B 25, 1759 (1982).

${ }^{15}$ J. M. Sanchez, F. Ducastelle, and D. Gratias, Physica A 128, 334 (1984).

${ }^{16}$ D. de Fontaine, Solid State Phys. 47, 33 (1994).

${ }^{17}$ L. G. Ferreira, S.-H. Wei, and A. Zunger, Phys. Rev. B 40, 3197 (1989).

${ }^{18}$ A. Zunger, in Statics and Dynamics of Alloy Phase Transitions, edited by P. E. A. Turchi and A. Gonis, NATO Advanced Studies Institute, Series B: Physics (Plenum Press, New York, 1994), pp. 361-419.

${ }^{19}$ D. B. Laks, L. G. Ferreira, S. Froyen, and A. Zunger, Phys. Rev. B 46, 12587 (1992).

${ }^{20}$ A. Zunger, L. G. Wang, G. L. W. Hart, and M. Sanati, Modell. Simul. Mater. Sci. Eng. 10, 685 (2002).

${ }^{21}$ G. L. W. Hart, V. Blum, M. J. Walorski, and A. Zunger, Nat. Mater. 4, 391 (2005)

${ }^{22}$ V. Blum, G. L. W. Hart, M. J. Walorski, and A. Zunger, Phys. Rev. B 72, 165113 (2005).

${ }^{23}$ A. van de Walle and G. Ceden, J. Phase Equilib. 23, 348 (2002).

${ }^{24}$ V. Blum and A. Zunger, Phys. Rev. B 70, 155108 (2004).

${ }^{25}$ J. Shao, J. Am. Stat. Assoc. 88, 486 (1993).

${ }^{26}$ K. Baumann, Trends Analyt. Chem. 22, 395 (2003).

${ }^{27}$ D. D. Richardson and J. Mahanty, J. Phys. C 10, 3971 (1977).

${ }^{28}$ L. G. Ferreira, S.-H. Wei, and A. Zunger, Int. J. Supercomput.
Appl. 5, 34 (1991).

${ }^{29}$ B. Predel, Phase Equilibria, Crystallographic and Thermodynamic Data of Binary Alloys, edited by O. Madelung, LandoltBörnstein, New Series, Group IV, Vol. 5, Pt. a (Springer, Berlin, 1991).

${ }^{30}$ C. Steiner, B. Schönfeld, M. J. Portmann, M. Kompatscher, G. Kostorz, A. Mazuelas, T. Metzger, J. Kohlbrecher, and B. Deme, Phys. Rev. B 71, 104204 (2005).

${ }^{31}$ E. Raub and W. Plate, Z. Metallkd. 48, 444 (1957).

${ }^{32}$ E. Raub and E. Roeschel, Z. Metallkd. 55, 320 (1964).

${ }^{33}$ L. Pauling, The Nature of the Chemical Bond and the Structure of Molecules and Crystals (Cornell University Press, Oxford, 1960).

${ }^{34}$ A. L. Allred and E. G. Rochow, J. Inorg. Nucl. Chem. 5, 264 (1958).

${ }^{35}$ M. C. Ball and A. H. Norbury, Physical Data for Inorganic Chemists (Longmans, London, 1974).

${ }^{36}$ This analysis, however, allows us to devise a qualitative electronegativity scale for the Rh-Pd-Ir-Pt quartet. Although there were no large electronegativity differences, we find that the electronegativities generally increase in the order $\mathrm{Rh}<\mathrm{Ir}<\mathrm{Pd}<\mathrm{Pt}$. These results parallel the findings of Watson and Bennett who based their electronegativity scale on characteristics of the puremetal densities of states. R. E. Watson and L. H. Bennett, Phys. Rev. B 18, 6439 (1978).

${ }^{37}$ A. R. Miedema, P. F. de Châtel, and F. R. Boer, Physica B \& C 100B, 1 (1980).

${ }^{38}$ A. Miedema, Philips Tech. Rev. 36, 217 (1976).

${ }^{39}$ It should be noted, however, that the energies predicted by the Miedema approach (with the exception of PdIr) are either below or only slightly above the theoretical resolution of the model.

${ }^{40}$ D. G. Pettifor, J. Phys. C 19, 285 (1986).

${ }^{41}$ A. Zunger, Phys. Rev. B 22, 5839 (1980).

${ }^{42}$ We used "SQS-8," an eight-atom special quasirandom structure best described as an $A_{2} B_{3} A_{2} B_{1}$ superlattice in the [113] direction. See S.-H. Wei, L. G. Ferreira, J. E. Bernard, and A. Zunger, Phys. Rev. B 42, 9622 (1990).

${ }^{43}$ G. Tréglia and F. Ducastelle, J. Phys. F: Met. Phys. 17, 1935 (1987).

${ }^{44}$ Z. W. Lu, S.-H. Wei, and A. Zunger, Europhys. Lett. 21, 221 (1993).

${ }^{45}$ L. G. Wang and A. Zunger, Phys. Rev. B 67, 092103 (2003).

${ }^{46}$ In our calculations, relativistic effects were treated within the scalar relativistic approximation.

${ }^{47}$ A. Zunger, S.-H. Wei, L. G. Ferreira, and J. E. Bernard, Phys. Rev. Lett. 65, 353 (1990). 